ORIGINAL RESEARCH

L. Khalid

M. Carone

N. Dumrongpisutikul

J. Intrapiromkul

D. Bonekamp

P.B. Barker

D.M. Yousem

\section{Imaging Characteristics of Oligodendrogliomas That Predict Grade}

\begin{abstract}
BACKGROUND AND PURPOSE: Oligodendrogliomas are tumors that have variable WHO grades depending on anaplasia and astrocytic components and their treatment may differ accordingly. Our aim was to retrospectively evaluate imaging features of oligodendrogliomas that predict tumor grade.
\end{abstract}

MATERIALS AND METHODS: The imaging studies of 75 patients with oligodendrogliomas were retrospectively reviewed and compared with the histologic grade. The presence and degree of enhancement and calcification were evaluated subjectively. rCBV and ADC maps were measured. Logistic linear regression models were used to determine the relationship between imaging factors and tumor grade.

RESULTS: Thirty of 75 (40\%) tumors enhanced, including 9 of 46 (19.6\%) grade II and 21 of 29 (72.4\%) grade III tumors $(P<.001)$. Grade III tumors showed lower ADC values compared with grade II tumors (odds ratio of a tumor being grade III rather than grade $\mathrm{II}=0.07 ; 95 \% \mathrm{Cl}, 0.02-0.25 ; P=.001$ ). An optimal ADC cutoff of $92510^{-6} \mathrm{~mm}^{2} / \mathrm{s}$ was established, which yielded a specificity of $89.1 \%$, sensitivity of $62.1 \%$, and accuracy of $78.7 \%$. There was no statistically significant association between tumor grade and the presence of calcification and perfusion values. Multivariable prediction rules were applied for $A D C<92510^{-6} \mathrm{~mm}^{2} / \mathrm{s}$, the presence of enhancement, and the presence of calcification. If either ADC $<92510^{-6} \mathrm{~mm}^{2} / \mathrm{s}$ or enhancement was present, it yielded $93.1 \%$ sensitivity, $73.9 \%$ specificity, and $81.3 \%$ accuracy. The most accurate $(82.2 \%)$ predictive rule was seen when either ADC $<92510^{-6} \mathrm{~mm}^{2} / \mathrm{s}$ or enhancement and calcification were present.

CONCLUSIONS: Models based on contrast enhancement, calcification, and ADC values can assist in predicting the grade of oligodendrogliomas and help direct biopsy sites, raise suspicion of sampling error, and predict prognosis.

ABBREVIATIONS: $\mathrm{Cl}=$ confidence interval; $\mathrm{rCBV}=$ relative cerebral blood volume; $\mathrm{ROC}=$ receiver operating characteristic analysis; $\mathrm{SE}=$ spin-echo; $\mathrm{WHO}=$ World Health Organization
$\mathbf{A}^{\mathrm{n}}$ mong the gliomas, oligodendrogliomas are the third most common, which account for 5\%-20\% of all glial tumors. ${ }^{1-3}$ They are slow-growing tumors, most often arising in the frontal lobe. ${ }^{3-5}$ More commonly found in the adult population, these tumors usually present in the fifth and sixth decades of life. ${ }^{4,6,7}$ Clinical presentation is usually seizures and headaches. $^{4,8}$

The WHO classification divides oligodendrogliomas histopathologically as low-grade (WHO II); high-grade or anaplastic (WHO III); anaplastic oligoastrocytomas with necrosis are classified as glioblastomas (WHO IV). ${ }^{9}$ Low-grade tumors may progress to high-grade tumors. However, this transformation is difficult to predict. $^{10}$

MR imaging is the best technique to diagnose these tumors. Studies have looked at MR imaging contrast enhancement, ${ }^{11-13}$ calcifications, ${ }^{14}$ and the use of diffusion and perfusion imaging to predict grade and anaplasia. ${ }^{15-17}$ However, no uniform predictive model presently exists for radiologic determination of the grade of these tumors. ${ }^{18}$

Received June 22, 2011; accepted after revision September 4

From the Russell H. Morgan Department of Radiology and Radiological Services (L.K., J.I., D.B., P.B.B., D.M.Y., N.D.), The Johns Hopkins Medical Institutions, Baltimore, Maryland; School of Public Health (M.C.), University of California, Berkeley, Berkeley, California; and Department of Radiology (N.D.), King Chulalongkorn Memorial Hospital, Pathumwan, Bangkok, Thailand.

Please address correspondence to David M. Yousem, MD, Department of Radiology, The Johns Hopkins Medical Institutions, 600 N Wolfe St, Phipps B100F, Baltimore 21287; e-mail: dyousem1@jhu.edu

http://dx.doi.org/10.3174/ajnr.A2895
The purpose of this study was to retrospectively evaluate imaging features of oligodendrogliomas that predict tumor grade. We also sought to assess whether contrast enhancement is seen as often as reported in the oligodendroglioma literature. In a similar fashion, we sought to assess whether tumoral calcification, perfusion values, and ADC values on MR imaging can be used in a predictive model for radiologic assessment of tumor grade. Tumor grade is important for directing biopsy sites, staging accurately, proving prognosis to patients, and raising suspicion of sampling error when discordant pathology and radiology studies are present.

\section{Materials and Methods}

After institutional research board approval and with Health Insurance Portability and Accountability Act compliance, 75 patients diagnosed with oligodendroglioma from the Johns Hopkins Hospital data base, who underwent MR imaging from 2004 to 2010, were retrospectively evaluated. The 75 patients had biopsy-confirmed oligodendroglioma, classified by an experienced neuropathologist according to the WHO criteria. Forty-six oligodendrogliomas were low-grade (WHO II), and 29 were high-grade (WHO III). Of the low-grade oligodendrogliomas, 44 were pure oligodendrogliomas and 2 were oligoastrocytomas. Of the high-grade tumors, 28 were anaplastic oligodendrogliomas and 1 was an anaplastic oligoastrocytoma.

MR imaging was performed at either $1.5 \mathrm{~T}$ or $3 \mathrm{~T}$. T1 SE, T2weighted, and FLAIR fast SE images were acquired. The sagittal T1weighted images were obtained with parameters as follows: TR range, 9.89-696 ms/TE range, 4.6-14 ms; matrix size from $192 \times 192$ to 


\begin{tabular}{llc}
\hline \multicolumn{2}{c}{ Table 1: Number of cases and their enhancement characteristics } \\
\hline Score & Degree of Enhancement & No. of Cases \\
\hline 1 & No enhancement & 45 \\
2 & Minimal enhancement & 17 \\
3 & Moderate enhancement & 8 \\
4 & Severe enhancement & 5 \\
\hline
\end{tabular}

$512 \times 196$; FOV from $190 \times 190 \mathrm{~mm}$ to $240 \times 240 \mathrm{~mm}$; range of section thickness/spacing of $1 / 1-5 / 7 \mathrm{~mm}$.

The axial T2-weighted images were obtained with a TR range, 2500-7000 ms/TE range, 83.136-112 ms; matrix size from $256 \times 184$ to $448 \times 335$; FOV from $159 \times 200 \mathrm{~mm}$ to $240 \times 240 \mathrm{~mm}$; range of section thickness/spacing of 2/2-5/5.

FLAIR scan parameters were the following: $\mathrm{TR}=6000, \mathrm{TE}=120$, $\mathrm{TI}=2000$, section thickness $=5 \mathrm{~mm}, \mathrm{FOV}=23 \mathrm{~cm}$, and matrix size $=256 \times 256$.

DWI was performed with the EPI sequence with a TR/TE range, 4900-10000 ms/80-133 ms; 5-mm thin contiguous sections; FOV of $220 \times 220 \mathrm{~mm}$ to $240 \times 240 \mathrm{~mm}$; and a matrix size of $96 \times 96$ to $192 \times 192$. Diffusion was measured in the 6 orthogonal directions with $2 \mathrm{~b}$-values $\left(0\right.$ and $\left.1000 \mathrm{~s} / \mathrm{mm}^{2}\right)$ with automated postprocessed ADC maps. Perfusion images by using a first-pass gadolinium technique were obtained with a $90^{\circ}$ flip angle and TR values between 1300 and $2000 \mathrm{~ms}$ and TE values between 40 and $60 \mathrm{~ms}$. Ten minutes after injection of gadopentetate dimeglumine $(0.1 \mathrm{mmol} / \mathrm{kg}$ of body weight) postcontrast T1-weighted SE images were acquired. All sequences had a section thickness of $5 \mathrm{~mm}$ and a section gap of $0-2.5$ $\mathrm{mm}$.

Two radiologists who were blinded to the histologic grade of the oligodendroglioma reviewed the studies independently. They reached a consensus regarding the presence or absence of tumor contrast enhancement. If they failed to reach consensus, a third neuroradiologist reader provided the deciding value for most opinions. The precontrast and postcontrast images were windowed and leveled identically to minimize any discrepancy in evaluating tumor contrast enhancement. Enhancement was scored as 1 (no enhancement), 2 (minimal enhancement), 3 (moderate enhancement), and 4 (severe enhancement) (Table 1). Disagreements were settled by consensus readings.

DWI sequences and ADC maps were reviewed, and ADC values were calculated. The most solid and lowest signal-intensity part of the tumor on ADC maps was marked as the ROI, and 4 different values of the intensity were noted within these regions, with the readers blinded to other pulse sequences. The area of lowest signal intensity on the ADC map was used, and there was no attempt to correlate this area with either 1) calcification, 2) enhancement, or 3) perfusion results. Care was taken in ensuring that all 4 ROIs were completely within the tumor in the 4 areas of lowest ADC. Each ROI was between 8 and 10 $\mathrm{mm}^{2}$. The T2-weighted and/or FLAIR images and/or enhanced T1weighted scans were used to accurately identify tumor location for the ADC maps. The lowest value of the 4 ROIs with its SD was recorded. We did not correlate or collocate the ADCs with the gadolinium enhancement, calcification, or perfusion data. We recorded the lowest values on the basis of visual inspection of ADC map and sampling of 4 ROIs, taking the lowest of these 4 values. These were then averaged for 1 common value between the 2 radiologists. If the value for 1 reader varied by $>20 \%$ from the second reader, a third reader measured the ADC value, and the average of the 2 closest values (corrected values) was used. Using a third reader was required in 19 (25.3\%) of the 75 cases. The mean coefficient of variation (difference between

\begin{tabular}{lcc}
\hline \multicolumn{3}{l}{ Table 2: Contrast enhancement in oligodendrogliomas } \\
\hline Tumor Grade & Enhancing & Nonenhancing \\
\hline III & 21 & 8 \\
II & 9 & 37 \\
Total & 30 & 45 \\
\hline
\end{tabular}

reads divided by the mean of the 2 reads) of the corrected $75 \mathrm{ADC}$ values was $8.4 \%$ (SD, $6.5 \%)$.

Measurements of rCBV were available for 20 cases. Dynamic susceptibility-weighted contrast-enhanced perfusion images were processed off-line by using in-house software, including a correction for blood-brain barrier permeability. ${ }^{19}$ Within the ROIs $\left(8-10 \mathrm{~cm}^{2}\right)$, the highest CBV value was noted and averaged. Perfusion values were selected separately and independently from the ROIs of the ADC values (ie, they were not in the same areas necessarily). When marking the ROI, we took great care to avoid regions of necrosis, calcification, cyst, or edema, aiming for the most solid part of the tumor. The final rCBV values used expressed the lesion CBV relative to $\mathrm{CBV}$ in the contralateral centrum semiovale white matter. Because this was a retrospective study, no standard protocol was used for imaging the tumors. Consequently, not all subjects included underwent perfusion studies.

The presence and absence of calcification were evaluated qualitatively on CT $(n=45)$ images. Preoperative CT scans were not available for all patients because of the lack of a standardized imaging protocol and the retrospective nature of the study. The presence of calcification and other imaging findings was compared with the histologic grade of tumor.

Prevalence proportions were estimated by using sample proportions, and corresponding CIs were constructed by using the score interval along with the Yates continuity correction. ${ }^{20,21}$ Logistic linear regression models were used to determine the relationship between given predictors and the outcome of interest; these provided odds ratios along with CIs and $P$ values obtained by inverting asymptotic $Z$-tests. ${ }^{20,21}$ Cross-validation was performed in each computation of the area under the ROC curve. When continuous predictors were dichotomized, the cutoff used was that which resulted in the classification rule with the greatest sum of sensitivity and specificity; this ensured a balanced trade-off between sensitivity and specificity. All computations were implemented by using the $\mathrm{R}$ statistical language (R Foundation for Statistical Computing, Vienna, Austria). ${ }^{22}$

\section{Results}

Of the 75 tumors, 30 (40.0\%; 95\% CI, 29.1\%-52.0\%) showed contrast enhancement. Enhancement was more common in grade III tumors, being observed in 9 of 46 (19.6\%; 95\% CI, $9.9 \%-34.4 \%)$ grade II tumors but 21 of $29(72.4 \%$; $95 \%$ CI, $52.5 \%-86.6 \%$ ) grade III tumors (Table 2). The odds of a tumor being grade III versus grade II were estimated to be 10.8 times greater when some enhancement was present (enhancement score, $\geq 2$ ) versus when none was found (enhancement, 1) $($ odds ratio $=10.8 ; 95 \% \mathrm{CI}, 3.6-32.2 ; P<.001)($ Fig 1$)$.

Tumors of grade III yielded lower ADC values, with a median ADC value of $1071 \times 10^{-6} \mathrm{~mm}^{2} / \mathrm{s}$ (first quartile: $1000 \times$ $10^{-6} \mathrm{~mm}^{2} / \mathrm{s}$; third quartile: $\left.1253 \times 10^{-6} \mathrm{~mm}^{2} / \mathrm{s}\right)$ in grade II tumors and of $908 \times 10^{-6} \mathrm{~mm}^{2} / \mathrm{s}$ (first quartile: $835 \times 10^{-6}$ $\mathrm{mm}^{2} / \mathrm{s}$; third quartile: $1021 \times 10^{-6} \mathrm{~mm}^{2} / \mathrm{s}$ ) in grade III tumors (Fig 2). ADC values were found to have relatively good predictive ability, with an area of 0.75 under the ROC curve. 

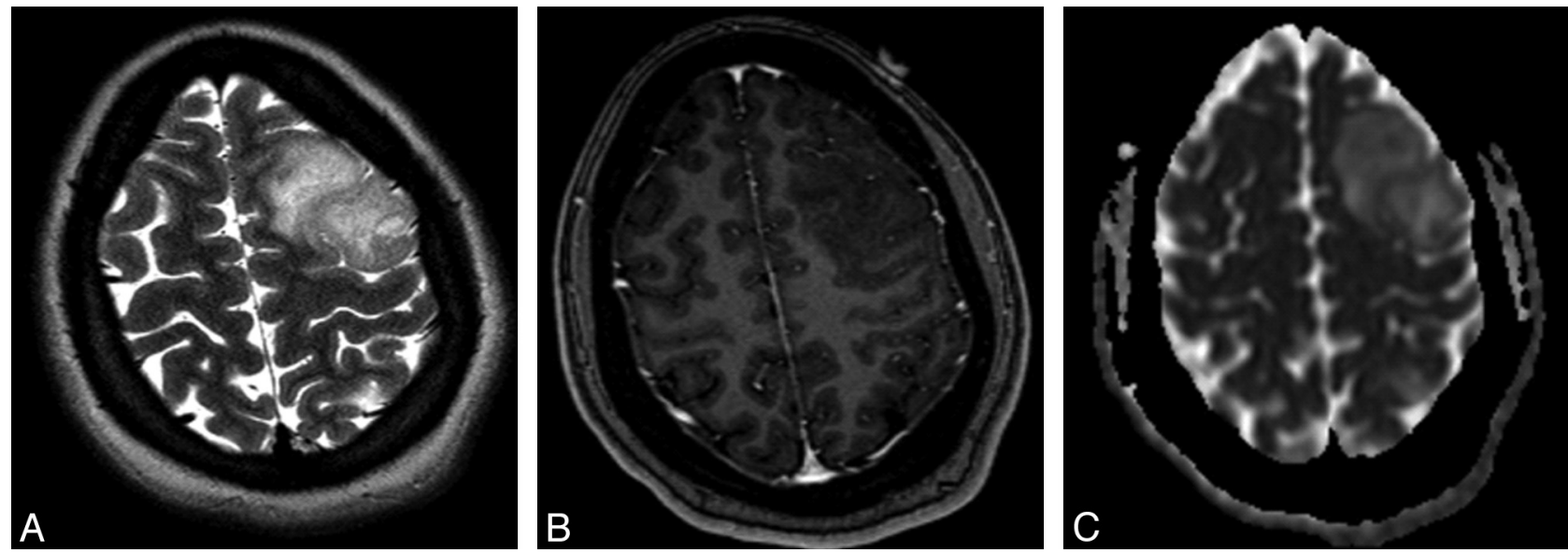

Fig 1. Grade II oligodendroglioma. A, Axial T2-weighted fast SE image shows a high-signal-intensity mass in left frontal lobe. $B$, Axial contrast-enhanced T1-weighted image shows no postcontrast enhancement within the left frontal lobe mass. C, Axial automated postprocessed ADC map yields a minimal ADC value of $946 \times 10^{-6} \mathrm{~mm}^{2} / \mathrm{s}$, above the $925 \times 10^{-6} \mathrm{~mm}^{2} / \mathrm{s}$ threshold.
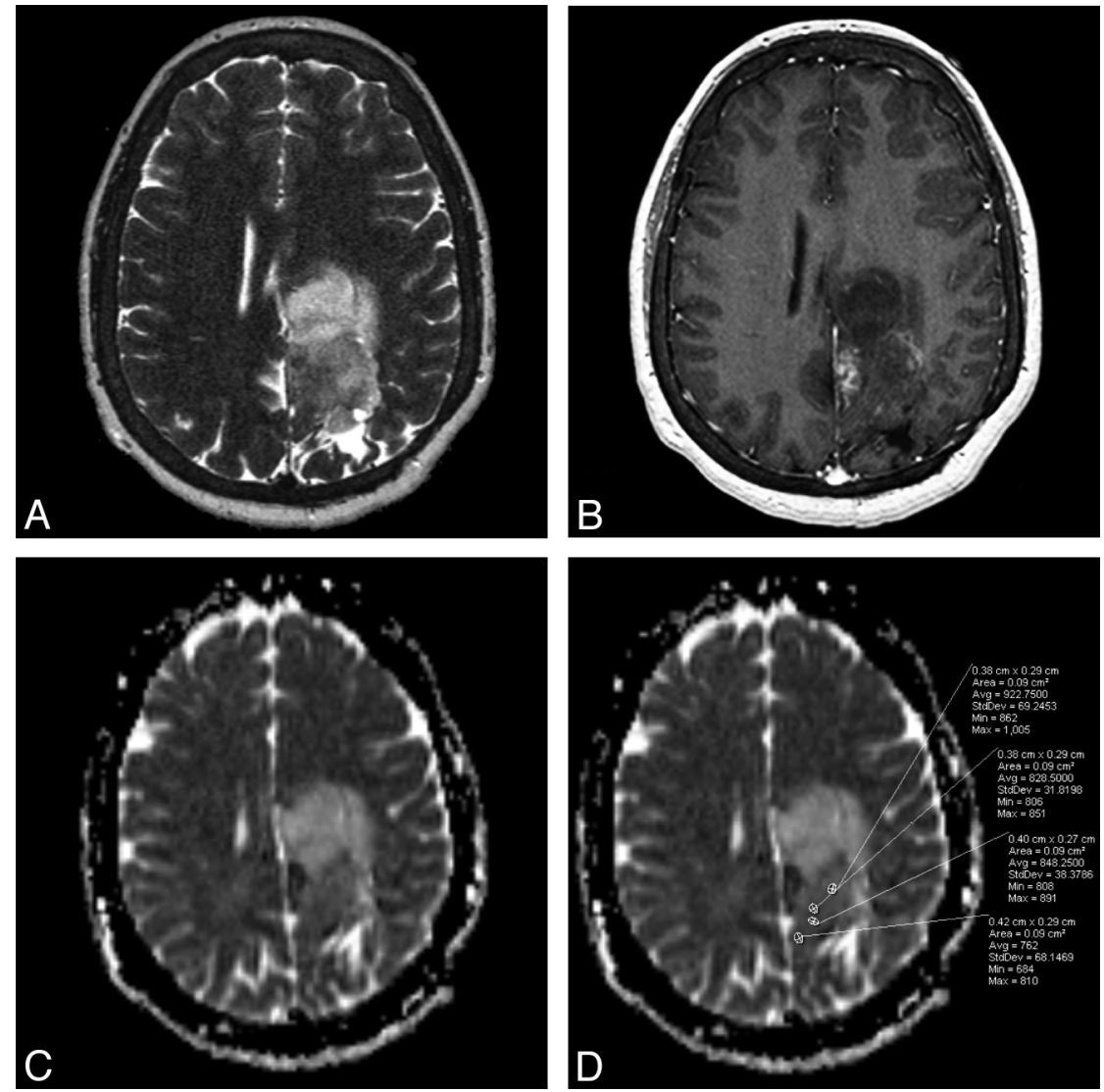

Fig 2. Grade III oligodendroglioma. A, Axial T2-weighted fast SE image shows a high-signal-intensity mass in left medial frontal and parietal lobes. B, Axial contrast-enhanced T1-weighted image shows minimal nodular post-contrast enhancement within the medial margin of the mass. C, Axial automated postprocessed ADC map shows low signal intensity along the posterior margin. D, As stated in "Materials and Methods," 4 ROls are placed in the areas visually assessed as the lowest in signal intensity on the ADC map. In this case, of the 4 ROls, the lowest ADC value is $762 \times 10^{-6} \mathrm{~mm}^{2} / \mathrm{s}$, which was recorded for this grade III oligodendroglioma, below the $925 \times 10^{-6} \mathrm{~mm}^{2} / \mathrm{s}$ threshold.

The optimal dichotomization cutoff value of ADC for classifying tumors as either grade II or III was identified as $925 \times$ $10^{-6} \mathrm{~mm}^{2} / \mathrm{s}$, with tumors with ADC values $\leq 925 \times 10^{-6}$ $\mathrm{mm}^{2} / \mathrm{s}$ being deemed grade III, and those with $>925 \times 10^{-6}$ $\mathrm{mm}^{2} / \mathrm{s}$, grade II. This classification rule for identifying grade III tumors resulted in a specificity of $89.1 \%$, sensitivity of $62.1 \%$, negative predictive value of $78.9 \%$, positive predictive value of $78.3 \%$, and accuracy of $78.7 \%$. Furthermore, tumors with a low ADC value $\left(\mathrm{ADC} \leq 925 \times 10^{-6} \mathrm{~mm}^{2} / \mathrm{s}\right)$ were estimated to have odds of being of grade III 13.4 times greater than tumors with a high $\mathrm{ADC}$ value $\left(\mathrm{ADC}>925 \times 10^{-6}\right.$ $\left.\mathrm{mm}^{2} / \mathrm{s}\right)($ odds ratio $=13.4 ; 95 \% \mathrm{CI}, 4.1-44.3 ; P<.001)$.

Calcification data were available for 45 of 75 cases. Calcification was more common in grade III tumors, being identified in 6 of 24 grade II tumors (25.0\%; 95\% CI, 10.6\%-47.1\%) and in 11 of 21 grade III tumors (52.4\%; 95\% CI, 30.3\%-73.6\%), 


\begin{tabular}{|c|c|c|c|c|c|c|}
\hline $\begin{array}{l}\text { Predict Grade III If } \\
\text { and Only If }\end{array}$ & Specificity & Sensitivity & $\begin{array}{c}\text { Negative Predictive } \\
\text { Value }\end{array}$ & $\begin{array}{c}\text { Positive Predictive } \\
\text { Value }\end{array}$ & Accuracy & $\begin{array}{c}\text { Specificity + } \\
\text { Sensitivity } \\
\end{array}$ \\
\hline A & $89.1 \%$ & $62.1 \%$ & $78.9 \%$ & $78.3 \%$ & $78.7 \%$ & $151.2 \%$ \\
\hline$E$ & $80.4 \%$ & $72.4 \%$ & $82.2 \%$ & $70.0 \%$ & $77.3 \%$ & $152.9 \%$ \\
\hline C & $75.0 \%$ & $52.4 \%$ & $64.3 \%$ & $64.7 \%$ & $64.4 \%$ & $127.4 \%$ \\
\hline$A+E$ & $95.7 \%$ & $41.4 \%$ & $72.1 \%$ & $85.7 \%$ & $74.7 \%$ & $137.0 \%$ \\
\hline$A+C$ & $91.7 \%$ & $38.1 \%$ & $62.9 \%$ & $80.0 \%$ & $66.7 \%$ & $129.8 \%$ \\
\hline$E+C$ & $87.5 \%$ & $52.4 \%$ & $67.7 \%$ & $78.6 \%$ & $71.1 \%$ & $139.9 \%$ \\
\hline$A$ or $E$ & $73.9 \%$ & $93.1 \%$ & $94.4 \%$ & $69.2 \%$ & $81.3 \%$ & $167.0 \%$ \\
\hline$A$ or $C$ & $75.0 \%$ & $81.0 \%$ & $81.8 \%$ & $73.9 \%$ & $77.8 \%$ & $156.0 \%$ \\
\hline E or C & $66.7 \%$ & $76.2 \%$ & $76.2 \%$ & $66.7 \%$ & $71.1 \%$ & $142.9 \%$ \\
\hline$A+E+C$ & $95.8 \%$ & $38.1 \%$ & $63.9 \%$ & $88.9 \%$ & $68.9 \%$ & $133.9 \%$ \\
\hline$A$ or $E$ or $C$ & $66.7 \%$ & $95.2 \%$ & $94.1 \%$ & $71.4 \%$ & $80.0 \%$ & $161.9 \%$ \\
\hline$A$ or $(E+C)$ & $83.3 \%$ & $81.0 \%$ & $83.3 \%$ & $81.0 \%$ & $82.2 \%$ & $164.3 \%$ \\
\hline$E$ or $(A+C)$ & $75.0 \%$ & $76.2 \%$ & $78.3 \%$ & $72.7 \%$ & $75.6 \%$ & $151.2 \%$ \\
\hline$C$ or $(A+E)$ & $75.0 \%$ & $61.9 \%$ & $69.2 \%$ & $68.4 \%$ & $68.9 \%$ & $136.9 \%$ \\
\hline$A+(E$ or $C)$ & $91.7 \%$ & $47.6 \%$ & $66.7 \%$ & $83.3 \%$ & $71.1 \%$ & $139.3 \%$ \\
\hline$E+(A$ or $C)$ & $87.5 \%$ & $61.9 \%$ & $72.4 \%$ & $81.3 \%$ & $75.6 \%$ & $149.4 \%$ \\
\hline$C+(A$ or $E)$ & $83.3 \%$ & $52.4 \%$ & $66.7 \%$ & $73.3 \%$ & $68.9 \%$ & $135.7 \%$ \\
\hline
\end{tabular}

${ }^{a}$ Based on the dichotomizations of $\operatorname{ADC}(A)$, enhancement $(E)$, and calcification (C).

though this difference was not statistically significant. The odds of a tumor being of grade III versus grade II were 3.3 times higher in the presence of calcification compared with absent calcification (odds ratio $=3.3 ; 95 \% \mathrm{CI}, 0.9-11.6 ; P=$ .063). As a classification rule for establishing tumor grade, with noncalcified and calcified tumors being deemed grades II and III, respectively, calcification had a specificity of $75.0 \%$, sensitivity of $52.4 \%$, negative predictive value of $64.3 \%$, positive predictive value of $64.7 \%$, and accuracy of $64.4 \%$.

Perfusion data were available for 20 of 75 cases. Tumors of grade III were found to exhibit lower perfusion values, with a median perfusion value of 2.12 (first quartile: 1.55 ; third quartile: 3.23 ) in grade II tumors and of 1.67 (first quartile: 1.39; third quartile: 21) in grade III tumors. The difference in perfusion values between groups was not statistically significant.

Classification rules involving several of the predictors discussed above were studied. Because of the large number of missing values, its lack of a significant association with tumor grade, and its overall poor value as a predictor, perfusion was excluded from multivariable analyses. The remaining 3 predictors (ADC value, enhancement, and calcification) jointly yielded an ROC curve with area of 0.88 , indicating that together they enjoyed very good predictive ability. The $17 \mathrm{mul}-$ tivariable prediction rules constructed by using the dichotomizations of ADC values, enhancement, and calcification were evaluated. These rules are provided in Table 3 along with their characteristics. Symbols A, E, and C refer to an ADC value $\leq 925 \times 10^{-6} \mathrm{~mm}^{2} / \mathrm{s}$, the presence of enhancement, and that of calcification, respectively. As such, the rule $\mathrm{A}+\mathrm{C}$ corresponds to predicting a tumor to be grade III if both an $\mathrm{ADC}$ value $\leq 925 \times 10^{-6} \mathrm{~mm}^{2} / \mathrm{s}$ and calcification were observed, for example, whereas the rule $\mathrm{E}+(\mathrm{A}$ or $\mathrm{C})$ corresponds to predicting grade III if both enhancement is observed and either the ADC value is $\leq 925 \times 10^{-6} \mathrm{~mm}^{2} / \mathrm{s}$ or calcification is present.

Nine of these rules could be eliminated because each of these was outperformed in all characteristics by at least 1 other rule (Table 3 ). Of the remaining 8 rules, 3 were identified as having particularly good overall performance, as character- ized by accuracy and the sum of sensitivity and specificity. Predicting a tumor to be grade III if either of the ADC value is $\leq 925 \times 10^{-6} \mathrm{~mm}^{2} / \mathrm{s}$ or enhancement is observed (A or E in Table 3) resulted in $73.9 \%$ specificity, $93.1 \%$ sensitivity, $94.4 \%$ negative predictive value, $69.2 \%$ positive predictive value, and $81.3 \%$ accuracy. This rule had the highest sum of sensitivity and specificity. Rendering this rule slightly more stringent by predicting a tumor to be grade III if either the ADC value is $\leq 925 \times 10^{-6} \mathrm{~mm}^{2} / \mathrm{s}$ or both enhancement and calcification are observed (A or $[\mathrm{E}+\mathrm{C}]$ in Table 3 ) resulted in a more balanced rule, with all measures of performance above $80 \%$. Specifically, this rule had a specificity of $83.3 \%$, a sensitivity of $81.0 \%$, a negative predictive value of $83.3 \%$, a positive predictive value of $81.0 \%$, and an accuracy of $82.2 \%$, the highest accuracy of all rules investigated.

\section{Discussion}

Grading oligodendrogliomas accurately is important because it has significant implications for the choice of technique used for the management of these tumors. Treatment for low-grade gliomas is primarily surgical resection and radiation therapy. ${ }^{13}$ Anaplastic oligodendrogliomas, on the other hand, show a favorable response to procarbazine, vincristine, lomustine, and temozolomide. ${ }^{23}$ Sampling errors and inconsistency in histopathologic grading of tumors can lead to over- or undertreatment of these tumors. Our predictive model tries to limit these errors in clinical management by providing a noninvasive tool with good predictive power to assess the grade of tumor.

Oligodendrogliomas have been reported to show frequent postcontrast enhancement on MR imaging. ${ }^{12}$ Gadolinium enhancement is suggested to be proportional to tumor vascularity. ${ }^{24}$ Most studies report that $50 \%-60 \%$ of oligodendrogliomas show contrast enhancement and that this feature is associated with high-grade tumors. ${ }^{11,24,25}$ Our results suggest that rate of enhancement of oligodendrogliomas is $40 \%$ (30/ 75). This is less than the reported $60 \%$ enhancement based on analysis of the literature. Among the enhancing tumors, a greater number of grade III tumors showed enhancement as 
opposed to grade II tumors, and this difference in enhancement is statistically significant. This result is different from the finding reported by White et $\mathrm{al},{ }^{25}$ who reported no difference in enhancement with grades of tumor. This significant difference in enhancement rates can help in differentiating lowgrade and high-grade tumors. As an independent predictor of tumor grade, we found it not to be very specific. A reason could be other factors influencing contrast enhancement rather than just the degree of neovascularity of the tumor, which correlates to the aggressiveness of tumor. Some studies have proposed a disruption of the blood-brain barrier as a contributing cause. $^{25}$

ADC values have shown high predictive power when it comes to differentiating high-grade and low-grade astrocytomas. Previous studies have used ADC maps on DWI to determine the tumor grade. ${ }^{15,26}$ An association between tumor cellularity and $\mathrm{ADC}$ has been proposed in the literature. ${ }^{26}$ Highgrade tumors are reported to have low ADC values, likely due to water restriction within these tumors secondary to a high nucleus-to-cytoplasm ratio and hypercellularity and higher ADC values in low-grade tumors. ${ }^{27}$ Our results support this by demonstrating a statistical difference between ADC values for grade III and grade II tumors. To establish the ADC value as a strong predictor, we propose a threshold value of $925 \times 10^{-6}$ $\mathrm{mm}^{2} / \mathrm{s}$ because this had the highest sum of sensitivity and specificity. ADC values may, therefore, be useful in assessing the grade of tumor, and ADC maps may also allow better localization of biopsy sites.

Calcification is a common finding in oligodendrogliomas. ${ }^{3}$ Calcifications were seen more frequently in grade III tumors rather than grade II tumors, though this was not statistically significant. A reason for this could be the limited sample size, and this can be overcome in future studies by increasing the number of cases. We found that calcification increases the power of our predictive model when used in conjunction with $\mathrm{ADC}$ and enhancement. Also the presence of calcification in an enhancing tumor is highly suggestive of a grade III oligodendroglioma. This will go on to increase the diagnostic confidence and help in guiding clinical management and support biopsy results.

Dynamic susceptibility contrast MR imaging data can be analyzed to generate various perfusion parameters in the brain, such as mean transit time and relative CBF and $\mathrm{rCBV}$. For the evaluation of tumors, the most commonly used parameter is rCBV, which is believed to reflect lesion vascular proliferation and attenuation. ${ }^{28}$ rCBV is often reported to be elevated in glial brain tumors compared with normal white matter, with rCBV values increasing with tumor grade, particularly for astroglial tumors. ${ }^{15,29}$ While some investigators have found increasing CBV with increasing grade in oligodendroglioma, ${ }^{30,31}$ others have found this not to be the case, ${ }^{32,33}$ with high rCBV often found in low-grade oligodendrogliomas, as was also observed in the current study. However, the $\mathrm{rCBV}$ results in the current study may not be particularly representative (and lack statistical significance) at least, in part, because of the relatively small number of subjects who underwent MR perfusion imaging in this retrospective study (20 of 75). It is possible that a larger study may find significant rCBV associations with grade in oligodendroglioma.

rCBV measurements in oligodendroglioma may still be clinically useful (eg, in terms of predicting treatment response or disease progression) even if they do not correlate with tumor grade. For instance, Whitmore et $\mathrm{al}^{30}$ have found increased rCBV in low-grade oligodendroglioma with $1 p / 19 q$ loss of heterozygosity; oligodendrogliomas with these mutations generally show a good response to chemotherapy. Also, another study has found strong negative correlations between $\mathrm{CBV}$ and time to progression in patients with low-grade gliomas (both astrocytomas and oligodendrogliomas), with low $\mathrm{CBV}$ indicating longer time to disease progression. ${ }^{34}$

As shown by multivariate association when the ADC threshold, enhancement, and calcification are used in conjunction, the predictive power of each measure increases significantly. Our predictive model based on the ADC value by using a cutoff of $925 \times 10^{-6} \mathrm{~mm}^{2} / \mathrm{s}$, the presence or absence of contrast enhancement, and calcification provides a promising noninvasive tool in guiding both surgical and clinical management of oligodendrogliomas. Using this model can guide physicians to accurate biopsy sites, reduce sampling error, and allow earlier decisions regarding management. The predictive models presented in this study are not powerful enough to replace histopathologic evaluation, which should still remain the criterion standard for diagnosing grade of tumor. The predictive models provide the advantage of time because they are easily obtainable and can influence decision-making while the tissue analysis results are pending.

The predictive power of our model can be improved by using a larger sample size in prospective studies with standardized protocols on magnets of similar field strength and the same manufacturer, for MR imaging of oligodendrogliomas. Because enhancement was judged subjectively, there is always a chance that subtle enhancement could have been missed. We tried to overcome this by minimizing interobserver variability and having a third radiologist act as the tiebreaker. In future studies, a more quantitative method to grade enhancement can be used to overcome this problem. Another limitation could be the placement of ROIs for measurement of ADC and $\mathrm{CBV}$ values, which was also performed subjectively because tumor borders are ill-defined. Although care was taken to place the ROIs entirely within the tumor, some variations in perfusion and ADC values could be secondary to inclusion of nontumorous areas. We tried to overcome this limitation by placing 4-5 ROIs within the tumor and averaging these to decrease the chance of an incorrect measurement. On the basis of incomplete 20-subject data, we cannot confidently rule out perfusion as a predictor of tumor grade. In future studies, more cases should be used to validate the predictive model, and a relationship between the predicted grade of tumor and survival rate can help establish the significance of these predictive parameters on MR imaging.

\section{Conclusions}

MR imaging contrast enhancement in oligodendrogliomas in the current series was less prevalent (40\%) than reported in the prior literature $(60 \%)$. Enhancement when used with the presence of calcification and/or an ADC threshold value of $925 \times$ $10^{-6} \mathrm{~mm}^{2} / \mathrm{s}$ can act as an accurate noninvasive predictor of tumor grade. This predictive model provides a reliable and rapid tool to better guide physicians when it comes to the localization of biopsy sites and can also facilitate earlier deci- 
sion-making and management. Histopathologic analysis of the tumor should still be considered the criterion standard for establishing tumor grade.

Disclosures: Netsiri Dumrongpisutikul_UNRELATED: Employment. King Chulalongkorn Memorial Hospital, Thai Red Cross Society, Grants/Grants Pending: King Chulalongkorn Memorial Hospital, Thai Red Cross Society. Peter Barker-UNRELATED: Grants/Grants Pending: Philips Healthcare.

\section{Acknowledgments}

We acknowledge the effort and contributions of Gloria Vila and John Boitnott for providing us with the list of cases for our study.

\section{References}

1. Claus EB, Black PM. Survival rates and patterns of care for patients diagnosed with supratentorial low-grade gliomas: data from the SEER program, 19732001. Cancer 2006;106:1358-63

2. McCarthy BJ, Propp JM, Davis FG, et al. Time trends in oligodendroglial and astrocytic tumor incidence. Neuroepidemiology 2008;30:34-44

3. Koeller KK, Rushing EJ. From the archives of the AFIP: Oligodendroglioma and its variants: radiologic-pathologic correlation. Radiographics 2005; 25:1669-88

4. Shaw EG, Scheithauer BW, O'Fallon JR, et al. Oligodendrogliomas: the Mayo Clinic experience. J Neurosurg 1992;76:428-34

5. Engelhard HH, Stelea A, Mundt A. Oligodendroglioma and anaplastic oligodendroglioma: clinical features, treatment, and prognosis. Surg Neurol 2003;60:443-56

6. Dehghani F, Schachenmayr W, Laun A, et al. Prognostic implication of histopathological, immunohistochemical and clinical features of oligodendrogliomas: a study of 89 cases. Acta Neuropathol 1998;95:493-504

7. Earnest F 3rd, Kernohan JW, Craig WM. Oligodendrogliomas: a review of 200 cases. Arch Neurol Psychiatry 1950;63:964-76

8. Wislawski J. Cerebral oligodendrogliomas: clinical manifestations, surgical treatment and histological findings in seventy cases. Pol Med J 1970;9:163-72

9. Louis DN, Ohgaki H, Wiestler OD, et al. The 2007 WHO classification of tumours of the central nervous system. Acta Neuropathol 2007;114:97-109. Epub 2007 Jul 6.

10. Brasil Caseiras G, Ciccarelli O, Altmann DR, et al. Low-grade gliomas: sixmonth tumor growth predicts patient outcome better than admission tumor volume, relative cerebral blood volume, and apparent diffusion coefficient. Radiology 2009;253:505-12

11. Fortin D, Cairncross GJ, Hammond RR. Oligodendroglioma: an appraisal of recent data pertaining to diagnosis and treatment. Neurosurgery 1999;45: 1279-91, discussion 1191

12. Lee YY, Van Tassel P. Intracranial oligodendrogliomas: imaging findings in $\mathbf{3 5}$ untreated cases. AJR Am J Roentgenol 1989;152:361-69

13. Leonardi MA, Lumenta CB. Oligodendrogliomas in the CT/MR-era. Acta Neurochir (Wien) 2001;143:1195-203

14. Wu Z, Mittal S, Kish K, et al. Identification of calcification with MRI using susceptibility-weighted imaging: a case study. J Magn Reson Imaging 2009; 29: 177-82

15. Arvinda HR, Kesavadas C, Sarma PS, et al. Glioma grading: sensitivity, speci- ficity, positive and negative predictive values of diffusion and perfusion imaging. J Neurooncol 2009;94:87-96

16. Cha S, Tihan T, Crawford F, et al. Differentiation of low-grade oligodendrogliomas from low-grade astrocytomas by using quantitative blood-volume measurements derived from dynamic susceptibility contrast-enhanced MR imaging. AJNR Am J Neuroradiol 2005;26:266-73

17. Chaskis C, Stadnik T, Michotte A, et al. Prognostic value of perfusion-weighted imaging in brain glioma: a prospective study. Acta Neurochir (Wien) 2006;148: 277-85, discussion 285

18. Alvord EC Jr. Is necrosis helpful in the grading of gliomas? Editorial opinion. J Neuropathol Exp Neurol 1992;51:127-32

19. Maia AC Jr, Malheiros SM, da Rocha AJ, et al. Stereotactic biopsy guidance in adults with supratentorial nonenhancing gliomas: role of perfusion-weighted magnetic resonance imaging. J Neurosurg 2004;101:970-76

20. Agresti A. Categorical Data Analysis. Hoboken, New Jersey: Wiley-Interscience; 2002

21. Rosner B. Fundamentals of Biostatistics. Boston: Duxbury Press; 2006

22. R Development Core Team. R: A Language and Environment for Statistical Computing. Vienna, Austria: R Foundation for Statistical Computing; 2010

23. Law M, Oh S, Johnson G, et al. Perfusion magnetic resonance imaging predicts patient outcome as an adjunct to histopathology: a second reference standard in the surgical and nonsurgical treatment of low-grade gliomas. Neurosurgery 2006;58:1099-107, discussion 1099-107

24. Reiche W, Grunwald I, Hermann K, et al. Oligodendrogliomas. Acta Radiol 2002;43:474-82

25. White ML, Zhang Y, Kirby P, et al. Can tumor contrast enhancement be used as a criterion for differentiating tumor grades of oligodendrogliomas? AJNR Am J Neuroradiol 2005;26:784-90

26. Kono K, Inoue $\mathrm{Y}$, Nakayama $\mathrm{K}$, et al. The role of diffusion-weighted imaging in patients with brain tumors. AJNR Am J Neuroradiol 2001;22:1081-88

27. Sadeghi N, Camby I, Goldman S, et al. Effect of hydrophilic components of the extracellular matrix on quantifiable diffusion-weighted imaging of human gliomas: preliminary results of correlating apparent diffusion coefficient values and hyaluronan expression level. AJR Am J Roentgenol 2003;181:235-41

28. Maia AC Jr, Malheiros SM, da Rocha AJ, et al. MR cerebral blood volume maps correlated with vascular endothelial growth factor expression and tumor grade in nonenhancing gliomas. AJNR Am J Neuroradiol 2005;26:777-83

29. Law M, Yang S, Wang H, et al. Glioma grading: sensitivity, specificity, and predictive values of perfusion MR imaging and proton MR spectroscopic imaging compared with conventional MR imaging. AJNR Am J Neuroradiol 2003;24:1989-98

30. Whitmore RG, Krejza J, Kapoor GS, et al. Prediction of oligodendroglial tumor subtype and grade using perfusion weighted magnetic resonance imaging. J Neurosurg 2007;107:600-09

31. Spampinato MV, Smith JK, Kwock L, et al. Cerebral blood volume measurements and proton MR spectroscopy in grading of oligodendroglial tumors. AJR Am J Roentgenol 2007;188:204-12

32. Lev MH, Ozsunar Y, Henson JW, et al. Glial tumor grading and outcome prediction using dynamic spin-echo MR susceptibility mapping compared with conventional contrast-enhanced MR: confounding effect of elevated rCBV of oligodendrogliomas [corrected]. AJNR Am J Neuroradiol 2004;25:214-21

33. Xu M, See SJ, Ng WH, et al. Comparison of magnetic resonance spectroscopy and perfusion-weighted imaging in presurgical grading of oligodendroglial tumors. Neurosurgery 2005;56:919-26, discussion 919-26

34. Caseiras GB, Chheang S, Babb J, et al. Relative cerebral blood volume measurements of low-grade gliomas predict patient outcome in a multi-institution setting. Eur J Radiol 2010;73:215-20 